\title{
Intel's Technology Vision and Products for HPC
}

\author{
Paweł Gepner \\ Intel Corporation \\ Phone: +48 602414128 \\ childs3@llnl.gov
}

\begin{abstract}
Traditionally most hardware and software architectural innovations have come through High End Computing. Today, innovation moves up from the bottom (low-power) and down from the top (parallelization) but the High-End is still a main foundation of new ideas. What is in today's supercomputer will be in tomorrow's desktop. What Intel does for HPC and what is the Intel vision and product portfolio for HPC segment will be covered during the lecture.
\end{abstract}

